\title{
Intrathoracic lipoma masquerading as subclavian
}

\section{artery trauma}

\author{
P T Munro, D O’Driscoll, K Mahalingam
}

\begin{abstract}
A 58 year old man was admitted to the accident and emergency department following an industrial accident in which he sustained a three part fracture dislocation of his right humerus. Chest radiography revealed a large mass in the right upper hemithorax and, when the patient became hypotensive, an emergency thoracotomy was performed. The mass was found to be a massive intrathoracic lipoma. This case shows how preexisting intrathoracic lesions may be mistaken for subclavian or great vessel trauma following violent shoulder girdle injury. The differential diagnosis of traumatic and non-traumatic intrathoracic mass lesions in chest radiography should be considered carefully.
\end{abstract}

(f Accid Emerg Med 1996;13:287-288)

Key terms: lipoma; radiography; thoracotomy; trauma

\section{Case report}

A 50 year old man was admitted to the accident and emergency (A\&E) department of Cork University Hospital after his clothing had become entangled in the machinery of a dockside crane. Clinical and radiological examination revealed a segmental fracture of the right humerus with subglenoid dislocation of the humeral head. The patient had evidence of radial nerve damage but the axillary nerve was intact and the distal pulses were normal. Examination revealed abrasions about the upper anterior chest but no other substantive injuries were identified and the vital signs were all within normal limits. The chest radiograph revealed a large ovoid soft tissue density in the right upper hemithorax (figure). The costophrenic angles were clear and there were no other significant abnormalities. It was initially felt by the clinicians that these appearances represented an incidental lesion such as a lypoma or dermoid cyst. Repair of the humeral head went ahead but, following induction of anaesthesia, the patient developed persistent and alarming hypotension. It was feared that the previously noted right upper thoracic mass was in fact an injury of the great vessels or subclavian artery and a right posterolateral thoracotomy was undertaken. At thoracotomy, a benign lipoma $(14 \times 13 \times 5$ $\mathrm{cm}$ in dimension ) was excised without difficulty. There was no evidence of trauma to any intrathoracic structure or the subclavian vessels, and it appeared in retrospect that the persistent hypotension was in fact an

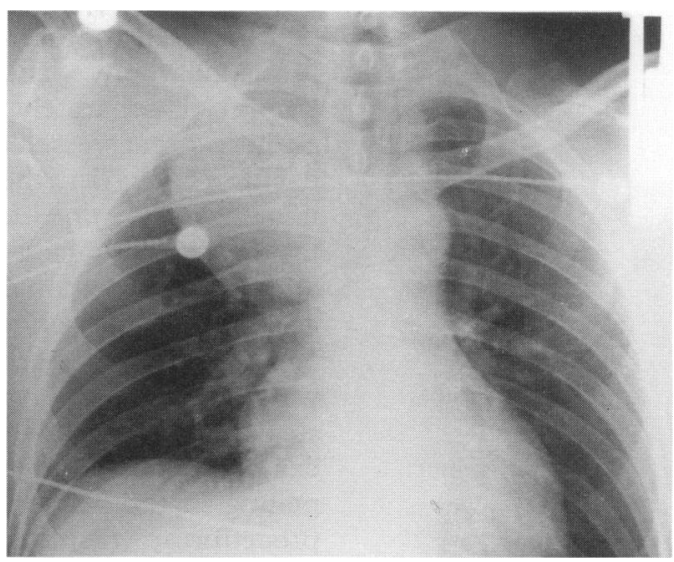

Chest radiograph showing a large soft tissue density in the right upper hemithorax.

anaesthetic complication. Subsequently, closed reduction of the right humeral head was performed and the fracture treated by RussellTaylor intramedullary nailing. The patient made an uneventful recovery and at review eight weeks later he had a virtually full range of movement in his right upper limb, without neurovascular deficit.

\section{Discussion}

Intrathoracic lipomas have been reported rarely as incidental findings on chest radiographs (with or without calcification ${ }^{1}$ ) in asymptomatic adult patients. Histologically they are composed of sheets of mature adipocytes separated by thin, incomplete fibrous septa containing a few blood vessels. In the chest, lipomas may be found within the lung substance or protruding into major bronchi. ${ }^{2}$ Malignant change is rare. In this case, given the severe damage to the upper limb it was clinically difficult to exclude the presence of contiguous injury to the subclavian artery (that is, pseudoaneurysm or associated haematoma). Blunt trauma to the subclavian vessels is associated with substantial mortality and morbidity, ${ }^{3}$ often due to embolic complications threatening the upper limb or cerebral circulation. The brachial plexus is frequently damaged in such injuries, either simultaneously or because of pressure effects from a subclavian artery false aneurysm. ${ }^{4}$

In retrospect, the initial chest radiograph of this patient combined with a clear understanding of the mechanism of injury (of the roller type) should have suggested a nontraumatic aetiology. However, the urgent nature of the clinical presentation obviously had its own dynamics. A non-traumatic cause 
for the intrathoracic mass could have been diagnosed on the basis of several radiological features: it was sharply delineated, there were no associated rib or scapular fractures, the costophrenic angles were clear of blood, and there was no shift or distortion of the mediastinum. A differential diagnosis of traumatic and non-traumatic lesions comparable with that on the chest radiograph can be found in the table. ${ }^{56}$ In practice, in a stable victim of trauma where there is such a mass lesion on the chest radiograph, a combination of computed tomography (CT), transthoracic ultrasonography, and angiography can be employed separately or in combination to exclude great vessel damage or intrathoracic haemorrhage. In the case described, such imaging was precluded by haemodynamic instability following induction of general anaesthesia. Although intraoperative angiography or ultrasonography could have excluded overt haemorrhage, surgical exploration was felt to be the most expedient option. Once subclavian and great vessel injury had been excluded and the mass excised, reduction and nailing of the humerus were relatively simple procedures, resulting in early functional recovery.

This case shows that unusual appearances on the chest radiograph following violent upper
Differential diagnosis of apical intrathoracic opacity (traumatic and non-traumatic)

\begin{tabular}{lrl}
\hline Traumatic & 1 & Haematoma (extra/intrathoracic) \\
& 2 & Aneurysm \\
& 3 & Postoperative (sympathectomy or plombage) \\
Non-traumatic & \\
Common: & 1 & Bronchogenic carcinoma (Pancoast tumour) \\
& 2 & Innominate artery aneurysm or unfolding \\
& 3 & Neurogenic tumour \\
& 4 & Retrosternal thyroid \\
Uncommon: 1 & Bronchogenic or tracheal cyst \\
& 2 & Cystic hygroma (lymphangioma) \\
3 & Lymphoma \\
4 & Other mediastinal tumour (including lipoma) \\
5 & Mesothelioma or other pleural tumour \\
& (including lipoma) \\
\hline
\end{tabular}

limb injury should prompt systematic radiological exclusion of significant contiguous vascular injury.

1 Sulzer MA, Foei R, Hollen EC, Theunissen PH, Massen FP. Lipoma of the external thoracic wall. Eur Resp 7 1994;7:207-9.

2 Corrin B. Tumours of fat. In: Symmers W St C, ed. Systemic pathology, vol 5, 3rd ed. Edinburgh: Churchill Livingston, 1990:907-8.

3 Pretre R, Hoffmeyer P, Bednarkiewicz M, Kursteiner K, Faidutti B. Blunt injury to the subclavian or axillary artery. F Am Coll Surg 1994;179:295-8.

4 Hansky B, Murray E, Minami K, Korfer R. Delayed brachial plexus paralysis due to subclavian pseudoaneurysm after clavicular fracture. Eur $\mathcal{F}$ Cardiothorac Surg 1993;7:497-8.

5 Gregson RHS. The mediastinum. In: Sutton D, ed. Textbook of radiology and imaging, vol 1, 5th ed. Edinburgh: Churchill Livingston, 1993:354-5.

6 Eisenberg RL. An atlas of differential diagnosis. London: Heinemann, 1988:128-33.

\title{
Road humps: accident prevention or hazard?
}

\author{
David Bowrey, Rhys Thomas, Rupert Evans, Peter Richmond
}

\begin{abstract}
Two cases of injury to passengers seated on public transport buses occurred after the vehicles had traversed road humps. While the potential dangers to seated bus passengers have been acknowledged in the Department of Transport literature, specific mention in medical reports is lacking. Should the United Kingdom follow the example set by the United States, where the placement of road humps on bus routes is avoided? (f Accid Emerg Med 1996;13:288-289)
\end{abstract}

Key terms: bus; injury; road hump

Road humps, or sleeping policemen, are devices placed in the path of travelling vehicles intended to be driven over at a comfortable predetermined speed, while causing increasingly more discomfort at higher speeds. Their main benefit lies in reducing vehicle speed, and thus improving traffic safety to residents and pedestrians in the neighbouring area. We report two cases of injury to passengers seated on public transport buses, the injuries occurring after the vehicles had crossed road humps. To the best of our knowledge these are the first reported cases of injury by this mechanism.

\section{Case 1}

A 49 year old female travelling on a double decker public transport bus was jolted upwards as the vehicle traversed a road hump and on landing back in her seat she experienced acute low back pain. Radiographs confirmed a crush fracture of her third lumbar vertebra (L3). Treatment comprised bed rest, analgesia, and a plaster jacket. One year after the injury she presented with further low back pain and paraesthesiae in both lower limbs. Magnetic resonance imaging (MRI) showed a posterior and right sided disc herniation at the level L2/3 causing root compression (figure). She underwent laminectomy and discectomy without undue complication.

Two years after the injury she continues to suffer from low back pain, and has been unable to return to her former employment. 